\title{
Predictors of adherence to different types and doses of supervised exercise during breast cancer chemotherapy
}

Kerry S Courneya ${ }^{1 *}$, Roanne J Segal ${ }^{2,3}$, Karen Gelmon ${ }^{4,5}$, John R Mackey ${ }^{1,6}$, Christine M Friedenreich ${ }^{7}$, Yutaka Yasui ${ }^{1}$, Robert D Reid ${ }^{8}$, Carolyn Proulx², Linda Trinh ${ }^{1}$, Lianne B Dolan ${ }^{4}$, Evyanne Wooding ${ }^{2,3}$, James R Vallerand ${ }^{1}$ and Donald C McKenzie ${ }^{4}$

\begin{abstract}
Background: Exercise is beneficial for breast cancer patients during chemotherapy but adherence to different types and doses of exercise is a challenge. The purpose of this study was to examine predictors of adherence to different types and doses of exercise during breast cancer chemotherapy in a multicenter randomized controlled trial.

Methods: Breast cancer patients in Edmonton, Vancouver, and Ottawa, Canada receiving chemotherapy $(\mathrm{N}=301)$ were randomized to a standard dose of 25-30 minutes of aerobic exercise (STAN), a higher dose of 50-60 minutes of aerobic exercise (HIGH), or a higher dose of 50-60 minutes of combined aerobic and resistance exercise (COMB). Predictors included demographic, medical, fitness, and quality of life variables. Exercise adherence was measured as the percentage of supervised exercise sessions completed.

Results: Overall adherence to the supervised exercise sessions was $73 \%(S D=24 \%$ ). In a multivariate regression model, six independent predictors explained 26.4\% ( $p<0.001)$ of the variance in exercise adherence. Higher exercise adherence was achieved by breast cancer patients in Vancouver $(p<0.001)$, with fewer endocrine symptoms $(p=0.009)$, randomized to STAN $(p=0.009)$, with fewer exercise limitations $(p=0.009)$, receiving shorter chemotherapy protocols $(p=0.015)$, and with higher $V_{2 \text { peak }}(p=0.017)$. Disease stage ( $p$ for interaction $\left.=0.015\right)$ and body mass index ( $p$ for interaction $=0.030$ ) interacted with group assignment to predict adherence. For disease stage, patients with stage I/lla disease adhered equally well to all three exercise interventions whereas patients with stage IIb/III disease adhered better to the STAN intervention than the two higher dose exercise interventions. For body mass index, healthy weight patients adhered equally well to all three exercise interventions whereas overweight patients adhered best to STAN and worst to COMB; and obese patients adhered best to STAN and worst to HIGH.
\end{abstract}

Conclusions: Determinants of exercise adherence in breast cancer patients receiving chemotherapy are multidisciplinary and may vary by the exercise prescription.

Keywords: Adherence, Cancer treatment, Cancer survivors, Determinants, Physical activity

\footnotetext{
* Correspondence: kerry.courneya@ualberta.ca

${ }^{1}$ Faculty of Physical Education and Recreation, University of Alberta,

E-488 Van Vliet Center, Edmonton, AB T6G 2H9, Canada

Full list of author information is available at the end of the article
} 


\section{Background}

Exercise during breast cancer chemotherapy improves physical functioning, fatigue, quality of life, and other symptoms [1]; and may even improve chemotherapy completion rate [2] and disease-free survival [3]. Moreover, higher doses of aerobic or combined exercise may provide additional benefits beyond a standard dose of aerobic exercise. In the Combined Aerobic and Resistance Exercise (CARE) Trial, we compared a thrice weekly standard dose of 25-30 minutes of moderate-to-vigorous intensity aerobic exercise (STAN) to a higher dose of 5060 minutes of aerobic exercise (HIGH) and a higher dose of 50-60 minutes of combined aerobic and resistance exercise $(\mathrm{COMB})$ in 301 breast cancer patients receiving chemotherapy. In the primary paper [4], we found several positive effects of the higher dose interventions compared to STAN for physical functioning, endocrine symptoms (e.g., hot flashes, night sweats), pain, and health-related fitness. In secondary papers, we also found that the higher dose interventions were more effective than STAN for managing sleep quality [5] and depressive symptoms in patients with clinical levels of depressive symptoms at baseline [6].

Unfortunately, adherence to exercise during breast cancer chemotherapy is challenging for any exercise intervention-let alone for higher dose interventions-due to the many side effects of treatments $[7,8]$. In the present study, we examined the predictors of exercise adherence in the CARE Trial. It is possible that breast cancer and/or chemotherapy-related variables may be unique determinants of exercise and/or they may amplify or nullify the predictive utility of other previously established determinants [9]. It is also possible that the determinants of higher dose exercise, or combined exercise, may be different than for standard dose exercise during breast cancer chemotherapy. Such data may be useful in developing targeted behavioral interventions to achieve better adherence to higher dose exercise interventions, thereby improving outcomes. To our knowledge, the CARE Trial is only the second trial to examine the determinants of exercise adherence during breast cancer chemotherapy, and the first to examine if the determinants of exercise adherence may vary based on the dose and type of exercise intervention.

Our hypotheses were based on the Supervised Trial of Aerobic versus Resistance Training (START) that compared aerobic exercise and resistance exercise to usual care in 242 breast cancer patients receiving chemotherapy [2]. In that trial, we found that higher cardiovascular fitness, lower body fat, more advanced disease stage, higher education, less depression, and being a non-smoker predicted better exercise adherence. Moreover, given the large sample size in the CARE Trial $(\mathrm{N}=301)$, we were able to explore potential interactions between these predictors and group assignment to directly test whether the predictors of exercise adherence during breast cancer chemotherapy differed by exercise type or dose.

\section{Methods}

\section{Setting and participants}

The main CARE Trial methods have been reported [4]. Briefly, the CARE Trial was a multicenter Canadian trial with sites in Edmonton, Ottawa, and Vancouver. Ethics approval was received from the Alberta Cancer Research Ethics Board and informed consent was obtained from all participants. Eligibility criteria included English or French speaking non-pregnant women $\geq 18$ years old with stage I IIIc breast cancer initiating adjuvant chemotherapy. Women were excluded if they had incomplete axillary surgery, transabdominal rectus abdominus muscle reconstructive surgery, significant health problems, or were not approved by their oncologist. Eligible participants were identified by their treating oncologist prior to chemotherapy.

\section{Randomization}

After all baseline assessments, participants were stratified by center and chemotherapy regimen (any Herceptin versus no Herceptin/any taxane versus no Herceptin/no taxane) and randomly assigned in a 1:1:1 ratio to STAN, $\mathrm{COMB}$, or $\mathrm{HIGH}$ using a computer-generated program. The allocation sequence was generated in Edmonton and concealed from the project directors who assigned participants to groups.

\section{Exercise training interventions}

The exercise training interventions have been previously described [4]. Briefly, participants exercised for the duration of their chemotherapy beginning within 1-2 weeks of starting chemotherapy and ending 3-4 weeks after chemotherapy. The STAN group were asked to follow the Physical Activity Guidelines for Americans [10] which have been endorsed for cancer survivors [11,12]. These guidelines recommend the equivalent of a minimum of 75 minutes/week of vigorous aerobic exercise spread over 3 days/week (i.e., 25-30 minutes/session). The HIGH group were asked to follow double the minimum guidelines of 150 minutes/week of vigorous aerobic exercise per week (i.e., 50-60 minutes/session). The COMB group were asked to complete the same aerobic exercise guideline as STAN plus a standard strength training program for 3 days/week consisting of two sets of 10-12 repetitions of 9 different strength exercises at $60-75 \%$ of their estimated 1 repetition maximum (i.e., about 50-60 minutes of combined exercise). The strength exercises were leg extension, leg curl, leg press, calf raises, chest press, seated row, triceps extension, biceps curls, and modified curl-ups. Aerobic exercise could be completed on a cycle ergometer, treadmill, elliptical, rowing ergometer, or combination. Initial exercise intensity was individualized but 
generally began at $55-60 \%$ of $\mathrm{VO}_{2 \text { peak }}$ and progressed to $70-75 \%$ of $\mathrm{VO}_{2 \text { peak }}$ by week 6 . Initial exercise duration was also individualized but generally began between 15-30 minutes/session and achieved 25-30 minutes/ session by week 4 (STAN and COMB) or 50-60 minutes/ session by week 6 (HIGH). Exercise trainers could modify the exercise prescription or progression based on the patient's response to the exercise and chemotherapy (i.e., toxicities). Unsupervised exercise was permitted but not encouraged.

\section{Assessment of exercise adherence}

The primary outcome measure for this study was the percentage of supervised exercise sessions completed, defined as the number of supervised exercise sessions attended divided by the number of supervised exercise sessions prescribed. The number of supervised exercise sessions prescribed was calculated from the time of randomization until three weeks after the final chemotherapy infusion, and therefore could vary by the length of the chemotherapy protocol as well as any chemotherapy delays. We did not include unsupervised sessions in our estimate of exercise adherence because, while unsupervised sessions were permitted, they were not encouraged and only $12 \%$ of all sessions were unsupervised [4]. Moreover, we focused on attendance because patients achieved their prescription goal in terms of intensity and duration, or weight, sets and repetitions in over $90 \%$ of their supervised sessions. Exercise adherence data were recorded by the exercise trainers at every supervised session.

\section{Assessment of predictors}

Exercise predictors included standard demographic and cancer variables, health-related fitness variables, and patient-reported outcome variables. Demographic data were collected by self-report and consisted of age (years), marital status (not married versus married), education (six categories ranging from some high school to completed graduate school), annual family income (six categories ranging from $<\$ 20,000$ to $\geq \$ 100,000$ ), employment status (not employed full-time versus employed full-time), ethnicity (other versus white), and location/center (Ottawa versus Edmonton versus Vancouver). Cancer variables were collected from medical records and consisted of disease stage (I/IIa versus IIb/III based on comparing primarily "node negative" or local disease versus primarily "node positive" or regional disease), type of surgery (lumpectomy versus mastectomy), and several chemotherapy variables including taxane versus no taxane, anthracycline versus no anthracycline, Herceptin versus no Herceptin, FEC-D (5-fluorouracil, epirubicin, cyclophosphamide, docetaxel) versus no FEC-D, concurrent versus sequential taxane, and 4 cycle (12 week) versus $6+$ cycle $(18+$ week) protocols. Other medical data consisted of self-reported comorbidities
(0 versus $1+$ ) and self-reported exercise limitations (none/ a little versus somewhat/a lot/completely). Behavioral variables were collected from self-report and consisted of smoking behavior (nonsmoker versus smoker) and baseline exercise behavior coded as meeting or not meeting current aerobic or resistance exercise guidelines.

Health-related fitness assessments have been described elsewhere [4] and consisted of variables related to peak oxygen consumption $\left(\mathrm{VO}_{2 \text { peak }}\right)$, muscular strength and endurance, body weight and height to calculate body mass index, and dual x-ray absorptiometry for the assessment of whole body fat and lean tissue. Patient-reported outcomes have also been detailed elsewhere [4] and consisted of the Medical Outcomes Survey Short Form (SF)36 physical component scales, physical well-being, breast cancer symptoms, fatigue symptoms, taxane/neuropathy symptoms, and endocrine symptoms.

\section{Statistical analyses}

Our study was originally powered for its primary purpose of examining the effects of exercise on patient-reported physical functioning [4]. For the present analyses, the 301 patients provided $80 \%$ power (two-tailed alpha $<0.05$ ) to detect a small correlation between a predictor variable and exercise adherence of approximately $r=0.15$. For nominal variables with more than two categories, we created a priori dichotomies based on standard groupings where possible (e.g., married versus not married, employed versus not employed). In the case of nominal variables with more than two categories that could not be dichotomized (i.e., group assignment and location/center), we created dummy-coded variables to allow for inclusion in a multivariate regression analysis. All continuous variables were analyzed as such (e.g., health-related fitness and patient-reported outcomes) although for ease of interpretation we also present categorical variables based on either statistically-determined tertiles or clinically established cut-points. We initially analyzed all predictor variables using Pearson $r$ correlations. Variables that had statistically significant or borderline significant $(\mathrm{p}<0.10)$ univariate associations with exercise adherence were included in a stepwise multivariate regression analyses. We also tested 12 interactions between the categorical demographic/medical variables and group assignment using analyses of variance.

\section{Results}

Participant flow through the trial has been reported elsewhere [4]. Briefly, we randomized 301 of 728 (41\%) eligible patients between April 2008 and September 2011. Study participants had a mean age of 50 years $(\mathrm{SD}=9)$, $65 \%$ were married, $65 \%$ had completed university, $23 \%$ were obese, $68 \%$ had stage I/IIa disease, $57 \%$ received breast conserving surgery, 34\% received FEC-D, 30\% 
reported regular aerobic exercise at baseline, and 21\% reported regular resistance exercise at baseline. We obtained exercise adherence data on all 301 patients. The mean length of the exercise intervention was 16.4 weeks $(\mathrm{SD}=3.6)$ resulting in an average of $49(\mathrm{SD}=11)$ possible exercise sessions. Overall mean adherence to the supervised exercise sessions was $73 \%(\mathrm{SD}=24 \%)$.

\section{Predictors of adherence to supervised exercise}

There were 16 significant or borderline significant univariate associations between the predictors and exercise adherence which are summarized in Tables 1 (demographic/ medical/behavioral), 2 (health-related fitness), and 3 (patient-reported outcomes). Variables that did not predict adherence in univariate analyses included all demographic factors (i.e., age, marital status, education, income, and ethnicity); several cancer variables (i.e., type of surgery, receipt of taxanes, anthracyclines, or Herceptin); and the patient-reported outcomes of physical functioning, fatigue, and breast cancer symptoms.
The 16 significant or borderline significant univariate predictors were included in a stepwise entry regression analysis. In the multivariate model, six predictors entered the model and explained $26.4 \%(\mathrm{p}<0.001)$ of the variance in exercise adherence (Table 4$)$. The six significant independent predictors were location/center $(\beta=$ $0.41 ; p<0.001)$, endocrine symptoms $(\beta=0.14 ; p=0.009)$, group assignment $(\beta=-0.13 ; p=0.009)$, exercise limitations ( $\beta=-0.13 ; \mathrm{p}=0.009$ ), length of chemotherapy proto$\operatorname{col}(\beta=-0.12 ; \mathrm{p}=0.015)$, and $\mathrm{VO}_{2 \text { peak }}(\beta=-0.12 ; \mathrm{p}=$ $0.017)$. Higher exercise adherence was achieved by breast cancer patients in Vancouver, with fewer endocrine symptoms, randomized to STAN, with fewer exercise limitations, receiving shorter chemotherapy protocols, and with higher $\mathrm{VO}_{2 \text { peak }}$ at baseline.

\section{Interactions between predictor variables and group assignment}

Significant interactions between the predictor variables and group assignment were identified for disease stage

Table 1 Significant demographic, medical, and behavioral predictors of adherence to supervised exercise in the CARE Trial, 2008-2011, Canada

\begin{tabular}{|c|c|c|c|}
\hline & & Correlation $^{1}$ & ANOVA $^{2}$ \\
\hline Variable & $M \pm S D(\%)$ & $\mathrm{r} ; \mathrm{p}$ value & F; $p$ value \\
\hline Group assignment & & $-.13 ; 0.025$ & $3.1 ; 0.048$ \\
\hline Standard aerobic $(n=96)$ & $78 \% \pm 24 \%$ & & \\
\hline Combined $(n=104)$ & $71 \% \pm 23 \%$ & & \\
\hline High aerobic $(n=101)$ & $70 \% \pm 25 \%$ & & \\
\hline Location/Center & & $.42 ;<0.001$ & $37.8 ;<0.001$ \\
\hline Ottawa $(\mathrm{n}=84)$ & $63 \% \pm 27 \%$ & & \\
\hline Edmonton $(n=117)$ & $68 \% \pm 23 \%$ & & \\
\hline Vancouver $(n=100)$ & $88 \% \pm 13 \%$ & & \\
\hline Baseline Aerobic Exercise & & $.14 ; 0.015$ & $5.1 ; 0.024$ \\
\hline Not meeting guidelines $(n=210)$ & $71 \% \pm 25 \%$ & & \\
\hline Meeting guidelines $(n=91)$ & $78 \% \pm 21 \%$ & & \\
\hline Exercise limitations & & $-.10 ; 0.098$ & $5.8 ; 0.017$ \\
\hline Not at all/a little $(n=150)$ & $76 \% \pm 22 \%$ & & \\
\hline Somewhat/a lot/completely $(n=151)$ & $70 \% \pm 26 \%$ & & \\
\hline Comorbidities & & $-.04 ; 0.54$ & $4.0 ; 0.047$ \\
\hline $0(n=135)$ & $76 \% \pm 23 \%$ & & \\
\hline$\geq 1(n=166)$ & $70 \% \pm 24 \%$ & & \\
\hline Length of chemotherapy protocol & & $-.13 ; 0.031$ & $4.7 ; 0.031$ \\
\hline 12 weeks $(n=89)$ & $78 \% \pm 21 \%$ & & \\
\hline$\geq 18$ weeks $(n=212)$ & $71 \% \pm 25 \%$ & & \\
\hline FEC-D ${ }^{3}$ & & $-.14 ; 0.013$ & $6.2 ; 0.013$ \\
\hline No $(n=200)$ & $75 \% \pm 22 \%$ & & \\
\hline Yes $(n=101)$ & $68 \% \pm 27 \%$ & & \\
\hline
\end{tabular}

Note. ${ }^{1}$ Tested the association as a continuous variable. ${ }^{2}$ Tested the association as a predefined categorical variable. ${ }^{3} \mathrm{FEC}-\mathrm{D}=5$-fluorouracil, epirubicin, cyclophosphamide, docetaxel. 
Table 2 Significant health-related fitness predictors of adherence to supervised exercise in the CARE Trial, 2008-2011, Canada

\begin{tabular}{|c|c|c|c|}
\hline Variable & $M+S D(\%)$ & Correlation ${ }^{1}$ & $\begin{array}{l}\text { ANOVA }^{2} \\
\text { F. } p \text { value }\end{array}$ \\
\hline$\overline{\mathrm{VO}_{2 \text { peak, }}, \mathrm{ml} / \mathrm{kg} / \mathrm{min}}$ & & $16 \cdot 0006$ & 43.0015 \\
\hline & & & \\
\hline$<25(n=100)$ & $69 \% \pm 25 \%$ & & \\
\hline $25-30(n=92)$ & $72 \% \pm 24 \%$ & & \\
\hline$>30(n=109)$ & $78 \% \pm 23 \%$ & & \\
\hline Peak rating of perceived exertion (0-10) & & $.27 ;<0.001$ & $30.7 ;<0.001$ \\
\hline$<10(\mathrm{n}=195)$ & $68 \% \pm 25 \%$ & & \\
\hline $10(n=106)$ & $83 \% \pm 20 \%$ & & \\
\hline Peak respiratory exchange ratio & & $.31 ;<0.001$ & $17.9 ;<0.001$ \\
\hline$\leq 1.10(n=123)$ & $66 \% \pm 26 \%$ & & \\
\hline$>1.10(\mathrm{n}=178)$ & $78 \% \pm 21 \%$ & & \\
\hline Leg Strength, kg & & $.25 ;<0.001$ & $7.4 ; 0.001$ \\
\hline$<70(\mathrm{n}=111)$ & $67 \% \pm 27 \%$ & & \\
\hline $70-90(n=88)$ & $74 \% \pm 23 \%$ & & \\
\hline$>90(n=102)$ & $79 \% \pm 20 \%$ & & \\
\hline Body fat, $\%$ & & $-.20 ;<0.001$ & $2.8 ; 0.062$ \\
\hline$<34(\mathrm{n}=94)$ & $77 \% \pm 25 \%$ & & \\
\hline $34-42(n=109)$ & $74 \% \pm 23 \%$ & & \\
\hline$>42(n=98)$ & $68 \% \pm 24 \%$ & & \\
\hline Lean body mass, kg & & $.16 ;<0.001$ & $4.5 ; 0.012$ \\
\hline$<37(\mathrm{n}=84)$ & $67 \% \pm 27 \%$ & & \\
\hline $37-42(n=112)$ & $72 \% \pm 24 \%$ & & \\
\hline$>42(n=105)$ & $78 \% \pm 21 \%$ & & \\
\hline
\end{tabular}

Note. ${ }^{1}$ Tested the association as a continuous variable. ${ }^{2}$ Tested the association as a predefined categorical variable.

Table 3 Significant patient-reported outcome predictors of adherence to supervised exercise in the CARE Trial, 2008-2011, Canada

\begin{tabular}{|c|c|c|c|}
\hline Variable & $\mathrm{M} \pm \mathrm{SD}(\%)$ & $\begin{array}{l}\text { Correlation }^{1} \\
r ; p \text { value }\end{array}$ & $\begin{array}{l}\text { ANOVA }^{2} \\
\text { F; } p \text { value }\end{array}$ \\
\hline Physical well-being & & $.12 ; 0.036$ & $2.5 ; 0.081$ \\
\hline$<23(\mathrm{n}=98)$ & $69 \% \pm 24 \%$ & & \\
\hline $23-25.9(n=93)$ & $74 \% \pm 25 \%$ & & \\
\hline$\geq 26(n=110)$ & $76 \% \pm 24 \%$ & & \\
\hline Taxane/neuropathy symptoms & & $.17 ; .003$ & $3.2 ; 0.043$ \\
\hline$<61(n=83)$ & $68 \% \pm 25 \%$ & & \\
\hline $61-63(n=102)$ & $73 \% \pm 24 \%$ & & \\
\hline $64(n=116)$ & $77 \% \pm 23 \%$ & & \\
\hline Endocrine symptoms & & $.17 ; 0.003$ & $1.5 ; 0.21$ \\
\hline$\leq 65(n=104)$ & $70 \% \pm 24 \%$ & & \\
\hline $66-71(n=99)$ & $74 \% \pm 23 \%$ & & \\
\hline$>71(\mathrm{n}=98)$ & $75 \% \pm 25 \%$ & & \\
\hline
\end{tabular}

Note. ${ }^{1}$ Tested the association as a continuous variable. ${ }^{2}$ Tested the association as a predefined categorical variable.
( $\mathrm{p}$ for interaction $=0.015$ ) and body mass index ( $\mathrm{p}$ for interaction $=0.030)$. For disease stage (Figure 1$)$, patients with stage I/IIa disease $(n=204)$ adhered equally well to all three exercise interventions whereas patients with stage IIb/III disease $(n=96)$ adhered better to the STAN intervention than the two higher dose exercise interventions. For body mass index (Figure 2), healthy weight patients $(n=148)$ adhered equally well to all three exercise interventions whereas overweight patients $(n=83)$ adhered best to STAN and worst to COMB; and obese

Table 4 Significant multivariate predictors of adherence to supervised exercise in the CARE Trial, 2008-2011, Canada

\begin{tabular}{lll}
\hline Variable & Standardized beta $(\boldsymbol{\beta})$ & $\mathbf{p}$ value \\
\hline Location/Center & 0.41 & $<0.001$ \\
Endocrine symptoms & 0.14 & 0.009 \\
Group assignment & -0.13 & 0.009 \\
Exercise limitations & -0.13 & 0.009 \\
Length of chemotherapy protocol & -0.12 & 0.015 \\
$\mathrm{VO}_{2 \text { peak, }} \mathrm{ml} / \mathrm{kg} / \mathrm{min}$ & 0.12 & 0.017 \\
\hline
\end{tabular}




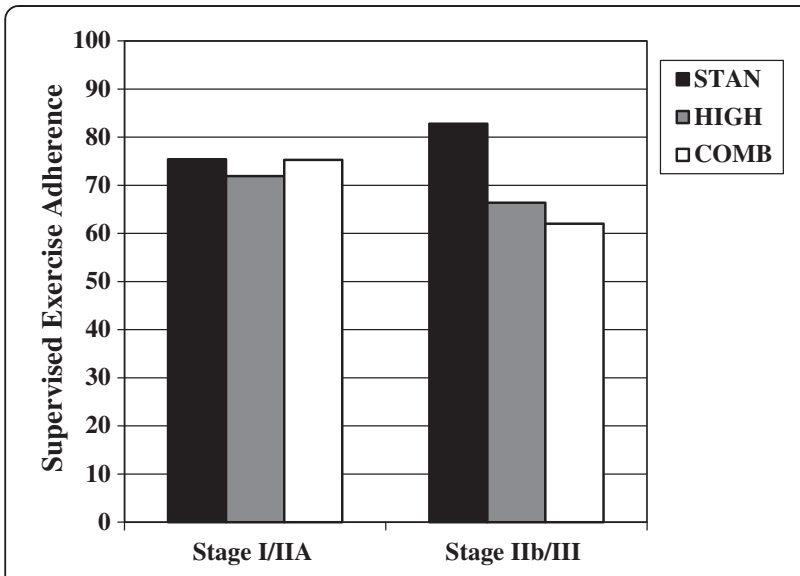

Figure 1 Significant interaction between disease stage and group assignment for predicting exercise adherence. STAN = standard aerobic exercise program; $\mathrm{HIGH}=$ high volume aerobic exercise program; $\mathrm{COMB}=$ combined aerobic and resistance exercise program.

patients $(n=70)$ adhered best to STAN and worst to HIGH.

\section{Discussion}

To our knowledge, only one other study has examined the predictors of exercise adherence during breast cancer chemotherapy [2]. Numerous studies have examined the cross-sectional correlates of exercise participation in breast cancer survivors, usually focusing on social cognitive variables and long term breast cancer survivors [13-19]. Moreover, several studies have examined the prospective correlates of exercise participation in breast cancer survivors $[20,21]$ or the predictors of adherence to a prescribed exercise program in long term breast cancer survivors $[22,23]$. The determinants of exercise adherence during breast cancer chemotherapy may be quite different than during survivorship because of the known toxicities

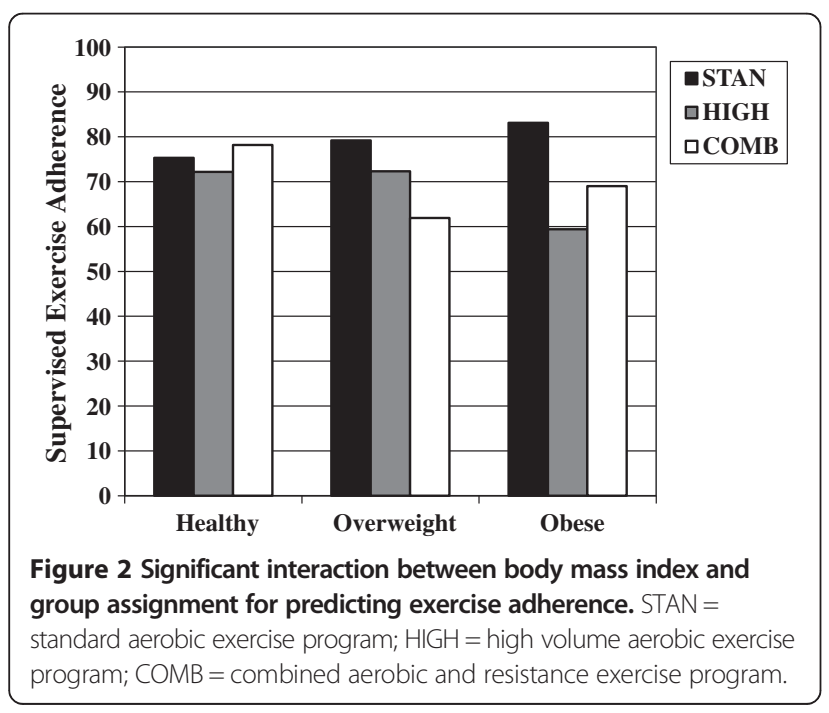

of these treatments. Moreover, none of these previous studies have examined adherence to different types and doses of exercise.

Overall adherence to supervised exercise in the CARE trial was $73 \%$. In our START trial involving breast cancer patients receiving chemotherapy, adherence to the aerobic exercise and resistance exercise interventions was about $70 \%$ each [2]. Adherence to the STAN intervention in the CARE Trial, which is comparable to the aerobic exercise intervention in the START Trial, was 78\% suggesting slightly better adherence in CARE despite the more difficult chemotherapy treatments containing taxanes. These adherence rates are within the range reported for other trials of exercise during cancer treatments [1]. Nevertheless, adherence in the CARE Trial was still not optimal and a better understanding of the determinants of adherence may inform behavior support interventions.

The strongest predictor of exercise adherence in the CARE Trial was the location/center at which the exercise was performed with Vancouver participants achieving higher adherence than Edmonton or Ottawa participants. We previously reported the same finding in our START Trial and offered several possible explanations [2]. One explanation was that Vancouver participants may have received more personal attention because the fitness center in Vancouver was only training CARE Trial participants whereas the Edmonton fitness center was training participants from multiple trials and the Ottawa fitness center was open to staff and patients not participating in clinical trials. This focus on breast cancer patients in Vancouver may have provided the women with more one-on-one attention to address individual needs. Moreover, we made a similar observation in our Alberta Physical Activity and Breast Cancer Prevention (ALPHA) Trial where we observed higher adherence to supervised exercise in Edmonton than Calgary [24]. We noted that the Edmonton training facility was exclusively for clinical trial participants whereas the Calgary training facility was a community-based facility open to the public [24]. Other possible explanations for site differences include: (a) the more moderate climate in Vancouver compared to harsher winters in Edmonton and Ottawa, (b) possible demographic differences in each city, (c) different referral patterns at each cancer center, and (d) travel time and convenience of accessing each fitness center. Future multicenter exercise trials should continue to report on adherence differences across sites.

Our trial also demonstrated that adherence to the STAN intervention was better than for the two higher dose exercise interventions. In some ways, this finding is not surprising given the many side effects of chemotherapy that may influence the amount of exercise completed during chemotherapy $[7,8]$. Nevertheless, it is unclear why doing more exercise during an exercise session (double the duration) would have an impact on the number of exercise 
sessions attended (frequency); especially given that fitness trainers instructed patients to complete only as much exercise as they were willing and able to do each session. One possible explanation is that when breast cancer patients were making a judgment about whether to attend their exercise session, they were factoring in the amount of time and/or anticipated difficulty of the session. Patients experiencing some fatigue, pain, hot flashes, nausea, or other symptoms that occurred during chemotherapy (i.e., after our baseline assessment of symptoms) might have felt that a $25-30$ minute exercise session would be tolerable but not a 50-60 minute aerobic or combined exercise session. Moreover, patients with busy lives and additional medical appointments might have felt that a 25-30 minute exercise session was more feasible than a 50-60 minute session. Based on our clinical observations, it is possible that 50-60 minutes of aerobic or combined exercise may be close to the "maximum tolerated dose" of exercise for breast cancer patients receiving current chemotherapy protocols.

Interestingly, the CARE trial showed that endocrine symptoms (e.g., hot flashes, night sweats) at baseline were negative predictors of exercise adherence in breast cancer patients. To our knowledge, the CARE trial is the first to report this association. It seems intuitive that these types of symptoms may interfere with a patient's ability and willingness to engage in exercise during chemotherapy. Nevertheless, the primary results of the CARE Trial actually showed that the higher dose exercise interventions were superior to STAN for managing endocrine symptoms [4]. Consequently, strategies to help breast cancer patients maintain exercise while experiencing such symptoms are needed (e.g., removing wigs, lighter clothing, cooler environments, personal fans).

Our trial also showed that exercise adherence during chemotherapy is predicted by the length of the chemotherapy protocol. Again, to our knowledge, the CARE Trial is the first to report that the length of the chemotherapy protocol may influence exercise adherence. It is well-known that the adverse effects of chemotherapy accumulate over the course of each cycle, and these accumulating side effects may interfere with exercise adherence $[7,8]$. Nevertheless, it is also possible that the lower adherence rate during longer chemotherapy protocols may simply reflect the difficulty of adhering to a longer exercise program. Unfortunately, we did not collect data on any toxicities during chemotherapy which might have directly informed this question. Consequently, these data only indirectly support the idea that chemotherapy toxicity may be the driving factor behind poorer exercise adherence to longer chemotherapy protocols. Regardless, additional support for patients on longer and/or more toxic chemotherapy protocols may be needed.
Patients with higher aerobic fitness and fewer functional problems also achieved better adherence. We observed the same finding in the START Trial [2]. It is possible that a minimum fitness level is needed to allow breast cancer patients to tolerate and respond to exercise training during chemotherapy. Future trials should determine if very unfit women can benefit from exercise during chemotherapy or whether it may be more prudent to initiate exercise in these women after adjuvant therapies are completed. In our paper reporting the sleep outcomes from the CARE Trial [5], we found that only higher fit breast cancer patients benefitted from the higher dose exercise interventions. The fact that baseline aerobic fitness is a predictor of exercise adherence is a dilemma for exercise researchers. Demonstrating the benefits of an exercise intervention is a precarious balance between enrolling participants who are most likely to benefit (i.e., generally those who are least fit) and enrolling participants who are most likely to adhere (i.e., generally those who are more fit).

We also identified two interesting interactions between the predictors and group assignment suggesting that different exercise interventions during chemotherapy may have different determinants of adherence. For body mass index, healthy weight patients adhered equally well to all three exercise interventions whereas overweight patients adhered best to STAN and worst to COMB; and obese patients adhered best to STAN and worst to HIGH. These data suggest that overweight and obese patients may struggle with higher dose exercise interventions. Several large studies have reported that higher body mass index is associated with lower exercise in cancer survivors [25-27]. Given that body mass index is one of the most consistent predictors of exercise adherence in cancer survivors, interventions targeting obese cancer survivors may be warranted, especially if higher dose exercise interventions are being contemplated.

For disease stage, patients with localized disease (stage I/IIa) adhered equally well to all three exercise interventions whereas patients with regional disease (stage IIb/III) adhered better to the STAN intervention than the two higher dose exercise interventions. In cross-sectional studies, the role of disease stage has been mixed with Irwin et al. [25] reporting a positive association between disease stage and exercise levels, Hong et al. [26] reporting a small negative association, and Milne et al. [27] reporting no association. In the START trial, we found that patients with more advanced disease achieved better adherence. The CARE Trial data are consistent with the START data showing that the more advanced stage patients had slightly better adherence to the STAN intervention (see Figure 2) which was comparable to the aerobic exercise intervention in START. However, the CARE Trial data show that these higher 
disease stage patients struggled with the higher dose exercise interventions.

It is important to briefly note the many variables in our study that did not predict exercise adherence either in univariate or multivariate analyses. Variables that predicted exercise adherence in univariate but not multivariate analyses included baseline aerobic exercise, comorbidities, FEC-D chemotherapy, peak rating of perceived exertion, peak respiratory exchange ratio, leg strength, lean body mass, \% body fat, physical well-being, and peripheral neuropathy symptoms. These variables may have relevance for exercise adherence and should be considered in future research. Variables that did not predict adherence, even in univariate analyses, included all demographic factors (i.e., age, marital status, education, income, and ethnicity); type of surgery or receipt of taxanes, anthracyclines, or Herceptin; and the patient-reported outcomes of physical functioning, fatigue, and breast cancer symptoms.

The strengths of our study include being the first study to prospectively examine the predictors of different types and doses of supervised exercise in breast cancer patients receiving chemotherapy, the assessment of many different potential predictors, the use of an objective measure of exercise adherence, the detailed cancer data that allowed us to examine the impact of several important chemotherapy variables, and the large sample size that allowed us to examine potential interactions between the predictors and the exercise interventions. One limitation of our study is the failure to examine predictors of long term exercise maintenance after a structured exercise program. We have previously reported predictors of exercise six months after supervised exercise during chemotherapy in breast cancer [28] and lymphoma patients [29]. In the CARE Trial, we will have data at six months, 1 year, and 2 years to examine this issue. Another limitation is that our predictors explained only $26 \%$ of the variance in exercise adherence, suggesting that other important factors may affect adherence. Some likely prospects include treatment toxicities and unexpected family events that occur after randomization which would require prospectively collecting such data. Finally, we conducted many statistical analyses which raises the possibility that some of the associations may be due to chance.

In summary, we examined predictors of adherence to three different exercise interventions in breast cancer patients receiving chemotherapy. Higher exercise adherence was achieved by breast cancer patients in Vancouver, with fewer endocrine symptoms, randomized to STAN, with fewer exercise limitations, receiving shorter chemotherapy protocols, and with higher $\mathrm{VO}_{2 \text { peak }}$ at baseline. Moreover, we identified two significant interactions suggesting that disease stage and body mass index may differentially predict adherence to the three different exercise interventions. Patients who were overweight/obese or had more advanced disease stage adhered equally well to the standard dose aerobic exercise intervention, but struggled with the higher dose exercise interventions. The present study provides an empirical basis for improving exercise adherence in breast cancer patients on chemotherapy, including adherence to higher dose exercise interventions which may provide additional benefits for symptom management [4] and even disease-free survival [3]. Additional research on the determinants of adherence to various exercise prescriptions during breast cancer chemotherapy is warranted.

\section{Competing interests}

The authors declare that they have no competing interests.

\section{Authors' contributions}

KSC, RJS, KG, JRM, CMF, YY, RD, and DCM made substantial contributions to conception and design, acquisition of data, analysis and interpretation of data, drafting of the manuscript, revising the manuscript, and gave final approval for publication. CP, LT, LB, and EW made substantial contributions to acquisition of data, revising the manuscript, and gave final approval for publication. JRV made substantial contributions to analysis and interpretation of data, revising the manuscript, and gave final approval for publication.

\section{Acknowledgments}

This work was supported by a grant from the Canadian Breast Cancer Research Alliance. KSC and YY are supported by the Canada Research Chairs Program. YY is also supported by the Alberta Innovates - Health Solutions. CMF is supported by a Health Senior Scholar Award from Alberta InnovatesHealth Solutions and through the Alberta Cancer Foundation's Weekend to End Women's Cancers Breast Cancer Chair. LT was supported by a Health Studentship from Alberta Innovates: Health Solutions.

\section{Author details}

${ }^{1}$ Faculty of Physical Education and Recreation, University of Alberta, E-488 Van Vliet Center, Edmonton, AB T6G 2H9, Canada. ${ }^{2}$ The Ottawa Hospital Cancer Center, Ottawa, Canada. ${ }^{3}$ Ottawa Hospital Research Institute, Ottawa, Canada. ${ }^{4}$ University of British Columbia, Vancouver, Canada. ${ }^{5}$ British Columbia Cancer Agency, Vancouver, Canada. ${ }^{6}$ Cross Cancer Institute, Edmonton, AB, Canada. ${ }^{7}$ Alberta Health Services, Calgary, Canada. ${ }^{8}$ University of Ottawa Heart Institute, Ottawa, Canada.

\section{Received: 29 March 2014 Accepted: 27 June 2014}

Published: 6 July 2014

\section{References}

1. Mishra SI, Scherer RW, Snyder C, Geigle PM, Berlanstein DR, Topaloglu O: Exercise interventions on health-related quality of life for people with cancer during active treatment. Cochrane Databse Syst Rev 2012, 15:1-459.

2. Courneya KS, Segal RJ, Gelmon K, Reid RD, Mackey JR, Friedenreich CM, Proulx C, Lane K, Ladha AB, Vallance JK, McKenzie DC: Predictors of supervised exercise adherence during breast cancer chemotherapy. Med Sci Sports Exerc 2008, 40:1180-1187.

3. Courneya KS, Segal RJ, McKenzie DC, Dong H, Gelmon K, Friedenreich CM, Yasui Y, Reid RD, Crawford JJ, Mackey JR: Effects of exercise during adjuvant chemotherapy on breast cancer outcomes [published online ahead of print March 13, 2014]. Med Sci Sports Exerc doi:10.1249/ MSS.0000000000000297.

4. Courneya KS, McKenzie DC, Mackey JR, Gelmon K, Friedenreich CM, Yasui Y, Reid RD, Cook D, Jespersen D, Proulx C, Dolan LB, Forbes CC, Wooding E, Trinh L, Segal RJ: Effects of exercise dose and type during breast cancer chemotherapy: multicenter randomized trial. J Natl Cancer Inst 2013, 105:1821-1831.

5. Courneya KS, Segal RJ, Mackey JR, Gelmon K, Friedenreich CM, Yasui Y, Reid RD, Jespersen D, Cook D, Proulx C, Trinh L, Dolan LB, Wooding E, Forbes CC, McKenzie DC: Effects of exercise dose and type on sleep quality in breast cancer patients receiving chemotherapy: a multicenter randomized trial. Breast Cancer Res Treat 2014, 144:361-369. doi:10.1007/s10549-014-2883-0. 
6. Courneya KS, McKenzie DC, Gelmon K, Mackey JR, Reid RD, Yasui Y, Friedenreich CM, Forbes CC, Trinh L, Jespersen D, Cook D, Proulx C, Wooding E, Dolan LB, Segal RJ: A multicenter randomized trial of the effects of exercise dose and type on psychosocial distress in breast cancer patients undergoing chemotherapy. Cancer Epidemiol Biomarkers Prev 2014, 23:857-864.

7. Courneya KS, McKenzie DC, Reid RD, Mackey JR, Gelmon K, Friedenreich CM, Ladha AB, Proulx C, Lane K, Vallance JK, Segal RJ: Barriers to supervised exercise training in a randomized controlled trial of breast cancer patients receiving chemotherapy. Ann Behav Med 2008, 35:116-122.

8. Courneya KS, Friedenreich CM, Quinney HA, Fields AL, Jones LW, Vallance JK, Fairey AS: A longitudinal study of exercise barriers in colorectal cancer survivors participating in a randomized controlled trial. Ann Behav Med 2005, 29:147-153.

9. Courneya KS: Physical activity and cancer survivorship: a simple framework for a complex field. Exerc Sport Sci Rev 2014, 42:102-109.

10. US Department of Health and Human Services: Physical Activity Guidelines for Americans. Hyattsville, MD: 2008. Available at http://www.health.gov/ paguidelines.

11. Schmitz KH, Courneya KS, Matthews C, Demark-Wahnefried W, Galvão DA, Pinto BM, Irwin ML, Wolin KY, Segal RJ, Lucia A, Schneider CM, von Gruenigen VE, Schwartz AL: American College of Sports Medicine roundtable on exercise guidelines for cancer survivors. Med Sci Sports Exerc 2010, 42:1409-1426.

12. Rock CL, Doyle C, Demark-Wahnefried W, Meyerhardt J, Courneya KS, Schwartz AL, Bandera EV, Hamilton KK, Grant B, McCullough M, Byers T, Gansler T: Nutrition and physical activity guidelines for cancer survivors. CA Cancer J Clin 2012, 62:243-274.

13. Blanchard CM, Courneya KS, Rodgers WM, Murnaghan DM: Determinants of exercise intention and behavior in survivors of breast and prostate cancer: an application of the theory of planned behavior. Cancer Nurs 2002, 25:88-95.

14. Courneya KS, Friedenreich CM: Utility of the theory of planned behavior for understanding exercise during breast cancer treatment. Psychooncology 1999, 8:112-122.

15. Charlier C, Van Hoof E, Pauwels E, Lechner L, Spittaels H, Bourgois J, De Bourdeaudhuij I: Treatment-related and psychosocial variables in explaining physical activity in women three weeks to six months post-treatment of breast cancer. Patient Educ Couns 2012, 89:171-177.

16. Charlier C, Van Hoof E, Pauwels E, Lechner L, Spittaels H, De Bourdeaudhui I: The contribution of general and cancer-related variables in explaining physical activity in breast cancer population 3 weeks to 6 months post-treatment. Psychooncology 2013, 22:203-211.

17. Phillips SM, McAuley E: Social cognitive influences on physical activity participation in long-term breast cancer survivors. Psychooncology 2013, 22:783-791.

18. Rogers LQ, Shah P, Dunnington G, Greive A, Shanmugham A, Dawson B, Courneya KS: Social cognitive theory and physical activity during breast cancer treatment. Oncol Nurs Forum 2005, 32:807-815.

19. Vallance JK, Lavallee C, Culos-Reed NS, Trudeau MG: Predictors of physical activity among rural and small town breast cancer survivors: an application of theory of planned behaviour. Psychol Health Med 2012, 17:685-697.

20. Emery CF, Yang HC, Frierson GM, Peterson LJ, Suh S: Determinants of physical activity among women treated for breast cancer in a 5-year longitudinal follow-up investigation. Psychooncology 2009, 18:377-386.

21. Loprinzi PD, Cardinal BJ, Si Q, Bennett JA, Winters-Stone KM: Theory-based predictors of follow-up exercise behavior after a supervised exercise intervention in older breast cancer survivors. Support Care Cancer 2012, 20:2511-2521.

22. Counreya KS, Blanchard CM, Laing DM: Exercise adherence in breast cancer survivors training for a dragon boat race competition: a preliminary investigation. Psychooncology 2001, 10:444-452.

23. Latka RN, Alvarez-Reeves M, Cadmus L, Irwin ML: Adherence to a randomized controlled trial of aerobic exercise in breast cancer survivors: the Yale exercise and survivorship study. J Cancer Surviv 2009, 3:148-157.

24. Courneya KS, Karvinen KH, McNeely ML, Campbell KL, Brar S, Woolcott CG, McTiernan A, Ballard-Barbash R, Friedenreich CM: Predictors of adherence to supervised and unsupervised exercise in the Alberta Physical Activity and Breast Cancer Prevention Trial. J Phys Act Health 2012, 9:857-866.
25. Irwin ML, McTiernan A, Bernstein L, Gilliland FD, Baumgartner R, Baumgartner K, Ballard-Barbash R: Physical activity levels among breast cancer survivors. Med Sci Sports Exerc 2004, 36:1484-1491.

26. Hong S, Bardwell WA, Natarajan L, Flatt SW, Rock CL, Newman VA, Madlensky L, Mills PJ, Dimsdale JE, Thomson CA, Hajek RA, Chilton JA, Pierce JP: Correlates of physical activity level in breast cancer survivors participating in the Womens Healthy Eating and Living (WHEL) Study. Breast Cancer Res Treat 2007, 101:225-232

27. Milne HM, Wallman KE, Guilfoyle A, Gordon S, Corneya KS: Self-determination theory and physical activity among breast cancer survivors. JSEP 2008, 30:23-38.

28. Courneya KS, Friedenreich CM, Reid RD, Gelmon K, Mackey JR, Ladha AB, Proulx C, Vallance JK, Segal RJ: Predictors of follow-up exercise behavior 6 months after a randomized trial of exercise training during breast cancer chemotherapy. Breast Cancer Res Treat 2009, 114:179-187.

29. Courneya KS, Stevinson C, McNeely ML, Sellar CM, Friedenreich CM, Peddle-McIntyre CJ, Chua N, Reiman T: Predictors of follow-up exercise behavior six months after a randomized trial of supervised exercise training in lymphoma patients. Psycho-Oncol 2012, 21:1124-1131.

doi:10.1186/s12966-014-0085-0

Cite this article as: Courneya et al:: Predictors of adherence to different types and doses of supervised exercise during breast cancer chemotherapy. International Journal of Behavioral Nutrition and Physical Activity $2014: 85$.

\section{Submit your next manuscript to BioMed Central and take full advantage of:}

- Convenient online submission

- Thorough peer review

- No space constraints or color figure charges

- Immediate publication on acceptance

- Inclusion in PubMed, CAS, Scopus and Google Scholar

- Research which is freely available for redistribution

Submit your manuscript at www.biomedcentral.com/submit
C) Biomed Central 